Muséologies

Les cahiers d'études supérieures

muséologies

\title{
Conversations et collaborations au musée : le cas de Tino Sehgal
}

\section{Isabelle Riendeau}

Volume 7, numéro 1, 2014

Le dialogue dans les musées d'art contemporain

URI : https://id.erudit.org/iderudit/1026654ar

DOI : https://doi.org/10.7202/1026654ar

Aller au sommaire du numéro

Éditeur(s)

Association Québécoise de Promotion des Recherches Étudiantes en Muséologie (AQPREM)

ISSN

1718-5181 (imprimé)

1929-7815 (numérique)

Découvrir la revue

Citer cet article

Riendeau, I. (2014). Conversations et collaborations au musée : le cas de Tino Sehgal. Muséologies, 7(1), 187-206. https://doi.org/10.7202/1026654ar

Tous droits réservés () Association Québécoise de Promotion des Recherches Étudiantes en Muséologie (AQPREM), 2014
Ce document est protégé par la loi sur le droit d'auteur. L'utilisation des services d'Érudit (y compris la reproduction) est assujettie à sa politique d'utilisation que vous pouvez consulter en ligne.

https://apropos.erudit.org/fr/usagers/politique-dutilisation/ 
Article huit

\section{Conversations et collaborations au musée : le cas de Tino Sehgal}

Isabelle Riendeau 
Titulaire d'un baccalauréat et d'une maîtrise en histoire de l'art de l'Université du Québec à Montréal, Isabelle Riendeau poursuit des études doctorales à la même université en muséologie, médiation et patrimoine. Ses recherches portent sur l'exposition et la médiation des pratiques dialogiques et participatives en contexte muséal. Elle travaille actuellement au Bureau d'art public de la Ville de Montréal comme agente de développement culturel et spécialiste de la médiation culturelle, en plus de collaborer à diverses publications. Au cours des quinze dernières années, elle a œuvré pour divers organismes culturels, enseigné l'histoire de l'art au collégial et réalisé des expositions à titre de commissaire indépendante. isabill@sympatico.ca 
Depuis les vingt dernières années, on constate une présence accrue, sur la scène de l'art contemporain, d'œuvres performatives, relationnelles, dialogiques ou sociales qui s'appuient sur les relations humaines et qui sollicitent directement le public en l'intégrant dans le processus créateur. L'idée d'un art sollicitant la participation du spectateur n'est toutefois pas nouvelle. Elle s'inscrit dans le prolongement historique de mouvements artistiques d'avant-garde des années 1960 et $1970^{1}$ et provient d'un besoin, de la part des artistes, de tisser des liens plus étroits avec la réalité et le public, de s'affranchir de l'objet et de l'institution contraignante qu'incarne le musée. Selon l'historien d'art Grant H. Kester, "The museum, with it's fusty, art historical associations, appeared ill equipped to provide a proper context for works that explored popular culture or quotidian experience ${ }^{2}$."

Marquant une rupture avec le musée, ces nouvelles formes artistiques se sont d'abord naturellement déployées en périphérie des lieux artistiques, soit dans l'espace public et la communauté $^{3}$. Toutefois, la présentation de l'exposition Traffic en 1996 au CAPC Musée d'art contemporain de Bordeaux par le conservateur Nicolas Bourriaud marque un tournant en muséologie et en art contemporain en offrant aux visiteurs non plus des objets à contempler, mais des espaces propices aux discussions et aux interactions. Dans la fin des années 1990, certains artistes en quête de légitimité et d'échanges sociaux effectuent un retour au

1 Nous référons aux formes d'art et aux mouvements influencés par Marcel Duchamp, le dadaïsme, le futurisme et l'avantgarde russe, dont Fluxus, l'Internationale situationniste, le Land Art, le Happening et la Performance. BISHOP, Claire. Artificial Hells. Londres/New York: Verso, 2012, chap. 1.

2 KESTER, Grant H. Conversation Pieces. Los Angeles:

University of California Press, 2004, p. 124.

3 Ces pratiques comprennent notamment l'art in situ (Daniel Buren) ou site specific (Miwon Kwon), les community-based projects (Miwon Kwon), les pratiques furtives (Patrice Loubier), les interventions en milieu urbain (Marie Fraser), l'art contextuel (Paul Ardenne), l'art engagé (Ève Lamoureux) ou socially engaged art (Pablo Helguera), l'art participatif(Claire Bishop). 4 BOURRIAUD, Nicolas. Esthétique relationnelle. Dijon: Les presses du réel, 1998, p. 31.

5 Nous référons notamment au Musée national des beauxarts du Québec (Massimo Guerrera, 2002-2003), au Musée d'art moderne de la Ville de Paris (Rirkrit Tiravanija, 2005), au Musée des beaux-arts du Canada (Flagrant délit - la per- musée, offrant ainsi un terreau fertile à l'émergence d'une esthétique relationnelle impulsée par Nicolas Bourriaud ${ }^{4}$. Depuis les années 2000, quelques musées d'art au Canada, aux États-Unis et en Europe ont relevé le défi de présenter des œuvres et des expositions impliquant la participation du visiteur ${ }^{5}$. Cependant, si la littérature consacrée aux pratiques participatives ou collaboratives concerne davantage les œuvres diffusées dans l'espace public pensons aux textes de Suzanne Lacy, Miwon Kwon, Grant H. Kester, Claire Bishop, Paul Ardenne, Nato Thompson, etc. -, les ouvrages théoriques sur les expositions présentées dans un cadre muséal demeurent rares.

Avec ces nouvelles formes d'art, le musée, habitué à exposer, à collectionner, à conserver des œuvres et à éduquer les visiteurs, deviendrait plus que jamais un lieu de rencontre entre les œuvres, les artistes, les professionnels et le public. Bien que la communication et l'interaction sociale fassent partie intégrante de la visite muséale ${ }^{6}$ et que le service de l'éducation prenne en charge le visiteur par toutes sortes de stratégies de médiation (visites, ateliers, conférences, etc.), ces pratiques vivantes ont fait de la relation et de la conversation le fondement même de l'œuvre ou de l'exposition ${ }^{7}$.

La présentation, par l'institution, d'un art axé sur la rencontre entraîne des bouleversements importants non seulement pour les professionnels, mais pour tous les intervenants en contact avec ces œuvres. Quels impacts leur

formance du spectateur, 2008), au Solomon R. Guggenheim de New York (Any Space Whatever, 2009), au Museum of Contemporary Art Chicago (Jeremy Deller, 2009), au Broad Art Museum de Lansing, Michigan (Jochen Gerz, 2012-

2013). Cette liste est évidemment non exhaustive.

6 Derking et Falk présentent les musées comme des environnements socioculturels où la conversation occupe une place prépondérante dans l'expérience muséale et l'apprentissage. FALK, John H. et Lynn D. DIERKING. Learning from Museums. Walnut Creek: AltaMira Press, 2000, p. 91-112.

7 Pour Kester (Conversation Pieces, op. cit., p. 90), les œuvres dialogiques doivent être considérées comme des processus de communication plutôt que comme des objets d'art. Référant à un texte de Reesa Greenberg, Glicenstein envisage pour sa part l'exposition comme un événement discursif, un modèle favorisant la prise de parole ou l'engagement d'un débat. GLICENSTEIN, Jérôme. L'art: une histoire d'exposition. Paris: Presses universitaires de France, 2009, p. 213. 
présentation a-t-elle sur les dynamiques relationnelles, les rôles de chacun des intervenants et sur les approches curatoriales et éducatives? Selon Jacques Rancière,

\section{Dans l'art " relationnel », la construction d'une situation indécise et éphémère appelle un déplacement de la perception, un passage du statut de spectateur à celui d'acteur, une reconfiguration des places [...] Le propre de l'art est d'opérer un découpage de l'espace matériel et symbo- lique. Et c'est par là que l'art touche à la politique $^{8}$.}

Dans ces conditions, comment un musée d'art contemporain avec le régime esthétique qui le caractérise - soit ses espaces neutres, sa mise à distance du visiteur et la construction d'un espace-temps séparé de la vie quotidienne conçoit-il l'exposition d'œuvres sociales et politiques axées sur le dialogue?

Le présent article aborde ces questions en regard d'une œuvre représentative de l'intégration de ces nouvelles formes d'art par le musée: This Progress de Tino Sehgal. Si cette œuvre incarne le " virage social " - pour paraphraser Claire Bishop ${ }^{9}$ - amené par les pratiques participatives dialogiques, la position de Sehgal est plus radicale en ce sens qu'il délaisse la matérialité et l'objet en mettant l'expérience humaine au premier plan. Les résultats que nous présentons dans cet article proviennent d'une étude de terrain réalisée à l'occasion de l'exposition Tino Sehgal présentée à l'hiver 2010 au Salomon R. Guggenheim Museum de New York ${ }^{10}$. Pour ce faire, nous avons privilégié deux méthodes: l'observation et

8 RANCIÈRE, Jacques. "L'esthétique comme politique ". Malaise dans l'esthétique. Paris: Galilée, 2004, p. 36-37.

9 BISHOP, Claire. "The Social Turn: Collaboration and its Discontents ". Artforum, février 2006. <http://artforum.com/ inprint $/$ issue $=200602 \&$ id $=10274>$ (consulté le 24 novembre 2010).

10 Cette étude de terrain réalisée à New York s'inscrit dans le cadre de notre thèse de doctorat sur la médiation des pratiques participatives et dialogiques.

11 L'artiste n'autorise aucune documentation de son travail, ce qui justifie que cet article soit illustré d'images du musée.

12 Tino Sehgal nomme ses œuvres Constructed Situations ou Staged Situations. l'entretien. Si l'observation nous a permis de mieux appréhender l'œuvre et ses défis, les entretiens réalisés avec un commissaire, deux guides, trois interprètes et l'artiste ont pallié la rareté des documents sur l'œuvre ${ }^{11}$. Pour comprendre les transformations qui s'opèrent au sein de l'institution, nous analysons le fonctionnement de cette exposition singulière, sa structure dialogique et discursive ainsi que le rôle de chacune des personnes impliquées dans le projet. Nous abordons ensuite l'important travail de coordination et de collaboration qu'a nécessité la présentation, par le Guggenheim, de cette situation construite ${ }^{12}$ d'envergure. Bien que cet article propose une analyse de This Progress, nous faisons également référence à This Situation, qui fut présentée au Musée d'art contemporain de Montréal au printemps 2013, afin de comparer ces deux œuvres fondées sur la participation et le dialogue ${ }^{13}$.

\section{Expérimenter This Progress de Tino Sehgal ${ }^{14}$}

27 février 2010. Nous franchissons les portes du musée Guggenheim où l'exposition Tino Sehgal est à l'affiche ${ }^{15}$. Dès notre arrivée, nous tentons d'emprunter les ascenseurs pour éviter la longue montée. Un employé du musée exerçant son travail dans l'anonymat - nous demande, sans plus d'explication, d'emprunter la rampe menant aux étages supérieurs. Une performance est en cours d'exécution; il s'agit de Kiss, une chorégraphie réalisée par des danseurs professionnels en hommage aux plus célèbres baisers de l'histoire de l'art ${ }^{16}$. En arrivant au premier palier, nous sommes accueillie par un enfant d'âge scolaire: «Hi, my name is... Welcome. This is a piece by Tino Sehgal. Would

13 Sehgal qualifie d'interprètes (interpreters) ou de joueurs (players) les gens qui exécutent ses pièces. Il s'agit de gens proches de l'artiste ou d'universitaires embauchés à la suite d'auditions.

14 Pour cette analyse, nous adoptons à la fois le point de vue du chercheur en muséologie et celui du visiteur.

15 Aucun cartel ou texte n'identifie l'œuvre ou l'artiste, suivant les consignes de ce dernier. Toutefois, le titre de l'œuvre est énoncé à voix haute par les interprètes.

16 Cette œuvre a été présentée dans plusieurs musées dont le Musée d'art contemporain de Montréal, du 19 mars au 28 avril 2013. 
you follow me? May I ask you a question? What is Progress? ${ }^{17}$ ". Cette phrase introduit This Progress, une situation qui consiste en une série de conversations entre quatre interprètes et des visiteurs autour du progrès. Pendant que nous répondons à l'enfant, l'ascension de la rotonde se poursuit. Quelques mètres plus loin, un adolescent nous rejoint en se présentant à son tour. L'enfant lui résume alors notre vision du progrès et nous poursuivons notre montée, pendant que l'adolescent nous invite à réfléchir davantage sur notre conception du progrès. Peu après, un trentenaire se joint à la conversation: interruption impromptue qui nous laisse perplexe et déstabilisée. Sans que nous en ayons pleinement conscience, l'adolescent s'est volatilisé. C'est en compagnie de ce troisième interprète que nous poursuivons la conversation et que nous progressons vers le sommet. Malgré le caractère insolite de la situation, nous nous sentons interpellée et prenons un réel plaisir à échanger avec cet inconnu. Au moment où nous passons devant une colonne, l'adulte nous cède poliment le passage et disparaît au moment où nous nous retournons pour lui répondre. C'est avec une consternation mêlée d'amusement - constatant que le même manège se répète chaque fois - que nous voyons un aîné venir vers nous, se présenter comme l'ont fait les autres avant lui. Une conversation animée s'engage autour d'une anecdote..., laquelle prend fin subitement lorsque notre interlocuteur s'arrête en disant: «This piece is called This Progress " avant de nous quitter. Vingt minutes s'écoulent. Déstabilisée, nous redescendons en croisant des gens seuls ou en groupe, en conversation animée avec des interprètes.

17 À l'exception de la phrase d'introduction à l'œuvre et celle qui la conclut, aucun scénario n'est prévu, bien que quelques consignes orientent les interprètes dans leur travail. (Entrevue avec Leland De La Durantaye, interprète, le 27 février 2010.) 18 La sélection des interprètes de This Situation s'est faite de manière similaire à celle de This Progress, soit de façon assez organique. Les gens pressentis pour jouer la pièce philosophes, sociologues, économistes, etc. - ont d'abord été sélectionnés par la commissaire Lesley Johnstone. Le producteur Asad Reza les a ensuite rencontrés individuellement pour leur parler du travail de Sehgal et les convaincre d'y participer.
L'expérience que nous propose This Situation, présentée en version bilingue au Musée d'art contemporain en 2013, est plus déroutante et évoque les discussions de salons entre intellectuels : six interprètes ${ }^{18}$ occupent la salle d'exposition, adossés aux murs vierges, à la manière de tableaux vivants. Lorsqu'un visiteur pénètre dans la salle, tous cessent leurs discussions et se tournent ensemble vers le visiteur pour l'accueillir et proclament en chœur : "Welcome to This Situation ». Ils prennent ensuite une longue et bruyante inspiration et se déplacent lentement, à reculons, le long du mur jusqu'au prochain " tableau "... tout en évitant les visiteurs. Ils demeurent silencieux jusqu'à ce qu'un interprète prenne la parole en déclamant une citation ${ }^{19}$ et en commençant toujours par: «In ..., somebody says... ». Après quelques instants de silence, les interprètes quittent leur position et une discussion s'engage autour de la citation, selon l'angle qu'ils ont choisi. Quelqu'un se tourne ensuite vers le nouvel arrivant et lui demande, en français: "Et vous, qu'en pensez-vous? " Le visiteur interpellé est invité à donner son point de vue sur la question et la discussion se poursuit, jusqu'à ce qu'une nouvelle personne entre dans la salle d'exposition. Tous s'interrompent et se tournent à l'unisson vers le nouveau venu. Le scénario se répète ainsi jusqu'à l'heure de fermeture du musée, donnant lieu à des conversations inachevées.

\section{Pour un nouveau paradigme au musée}

Les situations orchestrées par Tino Sehgal ébranlent les conventions et les fondements de l'institution muséale à plusieurs égards.

19 Il s'agit d'une des 20 à 40 citations philosophiques datant des années 1588 à 2005 apprises par cœur par les interprètes de la pièce et déclamées de façon aléatoire. Voir l'expérience relatée par Justin Erik Halldór Smith, un interprète de This Situation au Musée d'art contemporain de Montréal. <http://www.jehsmith.com/1/2013/04/interpreting-tino-sehgal.html> (consulté le 15 avril 2013). 
Puisque l'artiste n'autorise aucune forme de documentation ou de médiation de ses ouvres, elles n'existent que l'espace-temps de leur exposition et dans la mémoire de ceux qui les ont vues et expérimentées. Traduisant la double formation de l'artiste en danse et en économie politique, elles proposent, dans un cadre muséal, une expérience singulière et radicale, un espace de dialogue et d'échange. En dehors des ateliers de création ou autres activités éducatives, rares sont les occasions pour le public du musée de participer à l'élaboration d'une ouvre d'art. Comme toutes les pièces que l'artiste produit pour les musées, This Progress déjoue les conventions et les structures institutionnelles en mettant en scène l'expérience et l'histoire personnelles des gens plutôt qu'un objet physique à contempler ${ }^{20}$.

\section{L'émergence des pratiques participatives} qu'incarnent les œuvres de Tino Sehgal marque selon nous un changement de paradigme au musée. Bien que l'activité de perception confère au public un rôle actif face à l'œuvre ${ }^{21}$, nous passons d'un rapport esthétique et visuel à l'objet à un rapport relationnel, voire corporel, à l'art, où le sujet, autrefois regardeur ou observateur, occupe dorénavant une place prépondérante comme participant ou co-créateur ${ }^{22}$. Pour Kester, le degré d'implication du sujet dans l'esthétique traditionnelle est davantage lié à son appréciation ou son expérience physique de l'objet d'art, tandis que dans l'esthétique dialogique, c'est le rapport intersubjectif et la communication entre deux personnes qui génèrent le contenu de l'œuvre ${ }^{23}$. C'est donc dire que l'expérience muséale liée à l'art traditionnel (peinture, sculpture, photo, etc.) serait plus introspective pour le sujet, alors que dans l'art dialogique,

20 SPECTOR, Nancy. «Tino Sehgal: This Is Not an Object ». The Hugo Boss Prize 2006. Solomon R. Guggenheim Museum, Cantz: Ostfildern-Ruit, 2006, p. 89-93.

21 En 1957, Marcel Duchamp affirmait que le public contribuait au processus créateur en interprétant l'œuvre (<http://www.e-sociation.org/intra/index.php?option=com_co ntent\&view=article\&id=57:marcel-duchamp-qle-processuscreatifq\&catid $=43$ :grands-artistes $\&$ Itemid $=62>$ ). Depuis, plusieurs auteurs, dont Umberto Eco (L'œuvre ouverte, 1962), Roland Barthe (La mort de l'auteur, 1968), Jacques Rancière (Le spectateur émancipé, 2008) et André Gob (Le musée est-il une institution dépassée?, 2010) ont brisé le mythe du visiteur passif. celle-ci se consoliderait par les échanges interpersonnels. En abolissant la distance entre le visiteur et l'œuvre et les frontières entre l'art et le quotidien, ce que Rancière nomme la distance esthétique ${ }^{24}$, ces nouvelles pratiques artistiques transformeraient le musée et par le fait même l'espace d'exposition en une scène ou une plateforme d'action pour le visiteur. Entièrement immatérielles, This Progress et This Situation reposent sur l'interaction sociale entre les protagonistes (visiteur/interprète). Éphémères, elles n'existent que pour la durée de l'exposition et, bien que présentées en continu pendant les heures d'ouverture du musée, elles ne sont visibles et effectives qu'au moment où les gens entrent dans l'espace d'exposition et acceptent de discuter avec un interprète.

\section{Le fonctionnement et la structure de l'œuvre}

This Progress a été adaptée afin de répondre à la configuration particulière du lieu, occupant ainsi tout l'espace de circulation de la rotonde du Guggenheim. Modifiant les habitudes de circulation des usagers ${ }^{25}$, la pièce débutait au moment où ils empruntaient la rampe inclinée menant aux étages supérieurs et entraient en dialogue avec un premier interprète, et se terminait avec le quatrième interprète, lorsqu'ils atteignaient le dernier étage. Sehgal ne pouvait choisir un musée mieux approprié pour y présenter cette pièce, puisque l'architecture de la rotonde constitue une métaphore du progrès vue par la société occidentale: « "It's the perfect place" for this work, Sehgal said in an interview. "Every museum promotes the Western idea of progress, and this one epitomizes it" [...] It also suggests that progress, Sehgal says, is "nonlinear" $26 . "$

22 BISHOP, Artificial Hells, op. cit., p. 2.

23 KESTER, Conversation Pieces, op. cit., p. 112.

24 Voir le chapitre "Les paradoxes de l'art politique " dans RANCIÈRE, Jacques. Le spectateur émancipé. Paris : La Fabrique, 2008, p. 62-63.

25 L'architecte Frank Loyd Wright a défini les axes de circulation du musée afin que les gens commencent leur visite du haut jusqu'en bas de la rotonde.

26 STRICKLAND, Carol. «Is It Art? For Performance Artist Tino Sehgal, It's Immaterial ». The Christian Science Monitor. <http://csmonitor.com/layout/set/print/content/ view/print/278508> (consulté le 25 février 2010). 
S'appuyant entièrement sur la participation de gens de tous âges, une œuvre de cette ampleur requiert la présence de près de cent interprètes et constitue un défi important autant pour l'artiste que pour le musée. Chaque jour, plus de vingt personnes par segment de quatre heures étaient mobilisées pour interagir avec les visiteurs. Si plusieurs travaillaient en même temps, tous n'intervenaient pas au même moment puisque chacun d'eux avait un rôle particulier à jouer et entrait en scène, tel un acteur dans une pièce de théâtre, au moment qui lui était assigné. Il en était de même pour This Situation. Selon un interprète de l'exposition montréalaise, «There are rules governing what happens when, who moves when, and who says what when ${ }^{27}$."

This Progress comporte quatre sections distinctes, associées aux quatre catégories d'interprètes: les " enfants ", les " adolescents ", les "adultes " et les " aînés " (tableau 1). Le visiteur devait expérimenter successivement chacune des quatre zones dans l'ordre prévu, soit de bas en haut, pour comprendre le sens de la pièce. Au fur et à mesure qu'il progressait vers le haut, les interprètes devenaient plus âgés et la signification et le propos de l'œuvre se précisaient. Si les conversations avec les interprètes plus jeunes (enfants et adolescents) permettaient aux gens de partager puis d'élaborer leur propre conception du progrès, les échanges avec les interprètes plus âgés (adultes et aînés) se faisaient plus personnels, plus anecdotiques. Nous pourrions avancer l'hypothèse que notre vision de l'existence (une vision stéréotypée) se construit dès l'enfance, se définit et se nuance plus nous avançons en âge, alors que les expériences que nous vivons contribuent à nous définir. This Situation fonctionnait toutefois en boucle et ne comportait ni début ni fin. Le visiteur était libre d'arriver dans la pièce et de la quitter au moment où il le souhaitait:

27 Justin Erik Halldór Smith témoigne de son expérience comme interprète de This Situation présentée à Montréal. <http://www.jehsmith.com/1/2013/04/interpreting-tinosehgal.html> (consulté le 15 avril 2013).

28 Commentaire de l'interprète Stephen Rosenberg, cité dans HELGUERA, Pablo. "Is It Art or Is It Progress? ". Artword Salon. <http://www.artworldsalon.com/blog/2010/02/ is-it-just-art-or-is-it-progress> (consulté le 3 décembre 2012). tant que des gens demeuraient dans la salle, les conversations entre eux et les six interprètes se poursuivaient. Dès que de nouveaux visages entraient en scène, l'œuvre se modifiait, une nouvelle citation était prononcée et de nouvelles configurations devenaient possibles.

\section{Une situation aux contours indéfinis}

Une œuvre comme This Progress invite le participant à réfléchir sur sa conception du progrès. Puisque aucune autre indication n'est donnée à la question "What is Progress? ", celui-ci est libre de proposer sa définition. Elle peut être liée à philosophie, à l'économie, à la science, à la sociologie ou aux technologies - la réponse varie selon les participants. La majorité des personnes interrogées par les interprètes ont affirmé envisager le progrès comme une amélioration ou une évolution, prenant pour exemple la technologie et ses impacts sur leur vie ${ }^{28}$. Par le truchement de l'œuvre, cette conversation improvisée donnait une voix au public et, par le fait même, l'occasion de discuter de ses préoccupations concernant l'avenir en étant encouragé à le faire. Nous pourrions donc déduire que l'objectif de l'artiste n'était pas de faire émerger des définitions scientifiques sur le progrès ou de transmettre une vision unilatérale de ce qu'il représente, bien qu'il remette en question, dans son discours, la conception occidentale du progrès liée à la consommation ${ }^{29}$. Sehgal souhaitait plutôt provoquer, dans un cadre institutionnel, des discussions entre les gens et générer des moments de socialité comme alternative aux objets. Le sujet de l'œuvre, le progrès dans ce cas-ci, devenait un prétexte permettant aux visiteurs d'interagir avec d'autres dans un lieu voué à la contemplation d'œuvres d'art ${ }^{30}$. Il en était de même pour This Situation, où les sujets

29 Ces propos de Sehgal illustrent sa position face au progrès occidental: "In the last 200 or 300 years, the Western idea of progress is to transform the earth through human labor into material things, which we consume and derive income from and which make us happy. " STRICKLAND, "Is It Art or Is It Progress? ", op. cit. (consulté le 15 mars 2010). 30 Entrevue avec Sehgal, le 27 février 2010. 
Tableau 1

Tableau synthèse du fonctionnement de

This Progress

\begin{tabular}{|c|c|c|c|}
\hline $\begin{array}{l}\text { Ordre d'interaction } \\
\text { avec le visiteur }\end{array}$ & $\begin{array}{l}\text { Type d'interprète } \\
\text { sollicité }\end{array}$ & $\begin{array}{l}\text { Position physique de } \\
\text { l'interprète }\end{array}$ & $\begin{array}{l}\text { Rôle de l'interprète et } \\
\text { scénario de l'œuvre }\end{array}$ \\
\hline $1^{\mathrm{er}}$ & Enfant & $\begin{array}{l}\text { En haut de la rampe } \\
d^{\prime} \text { accès ( } 2^{\mathrm{e}} \text { étage) }\end{array}$ & $\begin{array}{l}\text { Introduit la pièce } \\
\text { et interroge le } \\
\text { visiteur sur sa vision } \\
\text { du progrès }\end{array}$ \\
\hline $2^{e}$ & Adolescent & $\begin{array}{l}2^{\mathrm{e}}, 3^{\mathrm{e}} \text { étages } \\
\text { (bas du parcours) }\end{array}$ & $\begin{array}{l}\text { Poursuit à partir de } \\
\text { la réponse fournie par } \\
\text { le visiteur }\end{array}$ \\
\hline $3^{e}$ & Adulte & $\begin{array}{l}3^{\mathrm{e}}, 4^{\mathrm{e}} \text { étages } \\
\text { (centre du parcours) }\end{array}$ & $\begin{array}{l}\text { Entre dans la } \\
\text { conversation dans un } \\
\text { rapport indirect et } \\
\text { raconte une anecdote }\end{array}$ \\
\hline $4^{e}$ & Aîné & $\begin{array}{l}5^{\mathrm{e}}, 6^{\mathrm{e}} \text { étages (sommet } \\
\text { de la rotonde) }\end{array}$ & $\begin{array}{l}\text { Livre une anecdote } \\
\text { liée au progrès et } \\
\text { conclut la pièce }\end{array}$ \\
\hline
\end{tabular}

Source: Isabelle Riendeau $\odot$. 
discutés par le truchement de citations philosophiques servaient surtout à susciter des réflexions et des questionnements.

Comme dispositifs à activer, les situations de Sehgal enjoignent le public à quitter sa position d'observateur et à aller au-delà de son appréciation traditionnelle et subjective de l'œuvre d'art pour interagir avec les interprètes. Interrogé et interpellé, il doit prendre position, élaborer sa pensée pour et avec l'autre (l'interprète), ou encore refuser d'y participer ${ }^{31}$. En s'adressant directement au public par le biais de ses interprètes, l'artiste le confronte à ses propres croyances et valeurs, démarche peu courante au musée en dehors des forums et des débats organisés. Au sujet de la place du visiteur dans l'œuvre, Sehgal affirme:

\section{Normally when you go into an exhibition you are confronted with something [...] In my case [...] the work basically asks the viewers, "What do you think?" and thereby also infers, what you do or what you say matters and will also change the course of this work ${ }^{32}$.}

Ce sont donc les réponses fournies par chacun qui influencent le déroulement de la pièce et alimentent la situation - bien que le thème général de la conversation (ou les citations dans le cas de This Situation) soit déterminé par l'artiste-, les interprètes se servant directement des répliques du visiteur pour construire le dialogue et articuler l'œuvre en devenir ${ }^{33}$. Autant This Progress que This Situation se présenteraient donc comme des créations inachevées, aux contours indéfinis, donnant lieu à de multiples possibilités selon les gens qui les nourrissent par leurs propos. Ce faisant, le musée pourrait, avec ces pratiques conversationnelles, se

31 Nous l'avons constaté sur le terrain lors de nos observations des œuvres This Progress et This Situation.

32 Citation de Tino Sehgal, en entrevue avec Hans Ulrich Obrist. VON HANTELMANN, Dorothea. " Objects and Situation in the Works of Tino Sehgal ". How To Do Things with Art. Zürich et Dijon: JRP Ringier et Les presses du réel, 2010, p. 166-167.

33 Entrevue avec Leland De La Durantaye, interprète, le 27 février 2010. concevoir comme un lieu de rassemblement et de dialogue, à l'image de l'agora ou du forum de l'Antiquité.

Ceux qui expérimentent les pièces à plusieurs occasions en découvriront à chaque fois une nouvelle facette, ce qui les rend dynamiques à la fois pour le public et pour les interprètes ${ }^{34}$. En permettant au visiteur de participer activement à l'élaboration des œuvres et en faisant appel à son histoire personnelle, Sehgal faciliterait-il l'appropriation de son travail ? C'est ce que croient les muséologues Lynn Derking et John Falk, qui affirment: "The sociocultural dimension of the Museum experience is an important component of the "contextual stamp" that enhaces people's ability to remember the experience $[\ldots]^{35}$ ». La conversation au musée comme mécanisme de construction de l'apprentissage, tout comme l'investissement des professionnels du musée et des artistes, seraient, selon ces chercheurs, des éléments à développer pour créer une expérience significative chez le visiteur au musée. Dans un texte sur les publics, l'historienne d'art Mieke Bal également souligne la nécessité pour le musée de réaliser des expositions qui engagent le visiteur et qui s'adressent à l'individu plutôt qu'au public en général ${ }^{36}$, ce que parviendrait à faire Sehgal en privilégiant un rapport spectateur/ interprète plutôt qu'un rapport matériel à l'art. Toutefois, si certains se sont sentis interpellés par l'œuvre et la discussion avec les interprètes, la structure rigide de la situation et du cadre muséal en a dérangé d'autres qui ont déploré le caractère artificiel de l'expérience ${ }^{37}$. Si This Progress instaure une relation plus intime entre l'interprète et le public par les conversations et les anecdotes qui s'échangent, This Situation apparait moins accessible au premier abord. En proposant une conversation davantage

34 Commentaire de Stephen Rosenberg, interprète, cité dans HELGUERA, " Is It Art or Is It Progress? ", op. cit. (consulté le 20 mars 2010).

35 FALK et DIERKING, Learning from Museums, op. cit., p. 109-110.

36 BAL, Mieke. "Le public n'existe pas ». In. CAILLET, Élisabeth et Catherine PERRET (dir.). L'art contemporain et son exposition. Tome 2. Paris : L'Harmattan, 2007, p. 10-35. 37 C'est ce qui ressort des commentaires formulés par certains visiteurs que nous avons interrogés lors de notre passage au musée Guggenheim en 2010. 
intellectuelle et formelle au sein d'un groupe fermé auquel n'appartient pas le visiteur, l'œuvre ne l'incite pas d'emblée à donner son avis, même s'il y est invité. Le ton officiel avec lequel les citations sont récitées, l'aspect hermétique et la blancheur de la salle d'exposition participent, à notre avis, à cette froideur qui se dégage de la pièce. Ce n'est qu'une fois le malaise initial surmonté que le plaisir de vivre l'œuvre et de prendre part aux conversations est possible, selon notre expérience et nos observations.

\section{La collaboration et la mise en œuvre d'une situation d'envergure}

La collaboration et le dialogue, omniprésents dans le travail de Tino Sehgal, s'incarnent également dans l'organisation de l'exposition et le travail de commissariat effectué par le musée. Réalisée à l'initiative de la conservatrice en chef Nancy Spector, en collaboration avec Nat Trotman et Katerine Brinson, commissaires, l'exposition du Guggenheim a nécessité une logistique importante et a requis un an de travail ${ }^{38}$. Les défis étaient nombreux pour l'équipe de commissaires et le musée a fait preuve d'audace en accueillant une œuvre radicale et singulière dans ce lieu touristique. Plusieurs étapes ont été nécessaires afin de mener à terme ce projet qui implique, pour le musée et l'artiste, de coordonner et de former plusieurs types d'intervenants avant que soit présentée la pièce.

En tant qu'auteur et metteur en scène de la situation, Sehgal définit les paramètres conceptuels et la structure de fonctionnement de l'œuvre dialogique, soit le thème, les rôles des quatre catégories d'interprètes, le scénario et le degré de liberté et de contrainte des interprètes et des visiteurs. Pour tous ses projets,

38 Entrevue avec Tino Sehgal à New York, le 27 février 2010.

39 Ce fut le cas pour This Situation présentée au Musée d'art contemporain de Montréal, confirme la commissaire Lesley Johnstone en entrevue le 24 avril 2013. Asad Reza a joué le rôle du producteur pour cette œuvre et coordonné l'ensemble du projet, avec la collaboration de la commissaire et de l'artiste demeuré seul à Berlin. il s'entoure d'une équipe de collaborateurs réguliers qui l'assistent dans la production et la logistique des expositions, le remplaçant lorsqu'il ne peut être présent ${ }^{39}$. Pour This Progress, l'artiste a travaillé en étroite collaboration avec l'équipe de commissaires en plus d'être sur place pour guider les interprètes tout au long de leur travail, tant lors des répétitions que de la présentation de l'exposition.

Quatre mois avant l'ouverture, l'artiste a entrepris, avec les commissaires, des auditions d'une grande ampleur afin de trouver des interprètes de quatre groupes d'âges (enfants, adolescents, adultes et aînés). L'objectif de cette démarche était de sélectionner des candidats curieux et instruits ayant la capacité d'entretenir des conversations sur divers sujets pendant une période de quatre heures consécutives. Ceux-ci ont d'ailleurs été, lors des entrevues, placés dans des situations qui ont permis d'évaluer leur capacité à improviser ${ }^{40}$.

Pour préparer son exposition et en vérifier l'efficacité, l'artiste a décidé de roder This Progress pendant l'exposition précédant la sienne, dans un musée bondé de gens et rempli d'œuvres. Pour ce faire, il a obtenu la collaboration d'amis et d'anciens collaborateurs qui ont endossé le rôle des interprètes auprès des visiteurs ${ }^{41}$. Pour l'exposition, la rotonde du musée a été entièrement vidée de ses œuvres d'art: une première en cinquante ans. Il en fut de même au Musée d'art contemporain où l'artiste tenait à éliminer toute interférence entre l'œuvre et le spectateur ${ }^{42}$.

Les premiers jours des expositions new-yorkaise et montréalaise, les interprètes, les commissaires et l'artiste/ le producteur ont dû procéder à certains ajustements afin de s'assurer que tout se déroule comme prévu et avec le plus de fluidité possible. L'artiste (lors de l'exposition

40 Entrevue avec Sam Sharp, interprète, le 27 février 2010.

41 Entrevue avec Leland De La Durantaye, interprète,

le 27 février 2010.

42 Il s'agit d'une requête de l'artiste, selon Lesley Johnstone, commissaire de l'exposition. 
à New York) et le producteur Asad Reza (lors de l'exposition à Montréal) observaient chaque jour les visiteurs et les interprètes en action, puis intervenaient a posteriori, lorsque la situation l'exigeait, pour apporter des précisions ou conseiller les interprètes quand ils éprouvaient des difficultés dans leur travail.

Cette phase préparatoire, préalable à la présentation de situations de cette complexité, illustre la pertinence d'une collaboration étroite entre tous les acteurs impliqués. Ces différentes étapes et constants allers-retours - essentiels dans le cas d'une œuvre processuelle - ont notamment permis à l'artiste et au musée de mettre en place les meilleures conditions possibles d'accueil, de présentation et de réception de l'œuvre.

\section{Une reconfiguration des rôles}

Accueillir une situation de Tino Sehgal fondée sur la participation et le dialogue implique une transformation des rôles des professionnels impliqués dans l'organisation, la présentation et la médiation de l'œuvre. Pour un commissaire, travailler avec des pratiques vivantes et des artistes en art contemporain suppose de prendre en compte leurs exigences, parfois inusitées, ainsi que leurs besoins, et de collaborer étroitement avec eux. La présentation de pièces dialogiques dans un cadre muséal entraîne selon nous deux changements majeurs dans la tâche du commissaire. D'une part, ce dernier agit plus comme coordonnateur de ressources humaines que responsable d'un corpus matériel et artistique. D'autre part, le commissaire n'agit plus seul: il partage son autorité et ses responsabilités avec l'artiste ou son producteur qui s'impliquent à toutes les étapes du processus, avant et pendant l'exposition. Interrogé sur son rôle, Nat Trotman du Guggenheim confirme que son travail se situe plus près de la

43 Entrevues réalisées avec Nat Trotman à New York le 27 février 2010 et avec Lesley Johnstone à Montréal le 24 avril 2013.

44 Au Québec, nous pensons notamment à Massimo Guerrera, Sylvie Cotton et Karen Élaine Spencer. gestion, alors que Lesley Johnstone, commissaire de l'exposition au Musée d'art contemporain de Montréal se considère davantage comme une intermédiaire entre les différents intervenants, soit le producteur de l'œuvre, les interprètes et les autres professionnels du musée ${ }^{43}$. Le fait que l'artiste et son producteur s'approprient en partie le rôle du musée forcerait ainsi ces professionnels à adapter leur façon de faire lorsqu'ils choisissent de présenter ce type d'expositions.

Doit-on en conclure que l'artiste ou son producteur détiennent plus d'autorité que quiconque au musée? Pas nécessairement. Si l'œuvre est conceptuellement réalisée par Tino Sehgal, son activation dans l'espace muséal, nous l'avons vu, est le fruit d'une responsabilité partagée par plusieurs intervenants, soit les interprètes et les visiteurs. À l'instar des artistes qui favorisent la participation du public dans leur travail ${ }^{44}$, Sehgal renonce en partie à son statut d'auteur lorsqu'il délègue l'interprétation de son œuvre. Nous sommes toutefois loin d'affirmer la mort de l'auteur ${ }^{45}$, puisque l'artiste élabore et transmet oralement les conditions de sa présentation ou de sa réitération. Comme l'écrivait justement Antoine Compagnon au sujet de l'écrivain, " On ne se débarrasse pas à si bon compte de l'auteur. Le lecteur a besoin d'un interlocuteur imaginaire, construit par lui dans l'acte de lecture, sans lequel la lecture serait abstraction vaine $[\ldots] »^{46}$.

Ce constat s'applique à l'œuvre de Sehgal où l'auteur occupe une place discrète, mais nécessaire. Il offre à l'interprète et au visiteur un cadre dans lequel ils peuvent évoluer, une structure qui permet à l'expérience de se réaliser et à l'œuvre de se manifester. Sans cette plateforme d'échange mise en place par l'auteur, y aurait-il toujours une œuvre, ou simplement un moment de socialité au musée?

45 BARTHES, Roland (1968). "La mort de l'auteur" (Publié à titre posthume, dans l'ouvrage Le bruissement de la langue. Paris: Seuil, 1984, p. 61-67).

46 COMPAGNON, Antoine. "Introduction: mort et résurection de l'auteur ". Fabula: la recherche en littérature. <http://www.fabula.org/compagnon/auteur1.php> (consulté le 28 mars 2013). 
Le succès des situations de Sehgal repose en grande partie sur l'activité des interprètes qui interagissent avec les visiteurs, d'où la nécessité de les choisir judicieusement. Leur travail les place dans une relation d'interdépendance avec le public, ce qui les oblige à être à l'écoute des participants et à faire preuve d'ouverture tout au long de la discussion. Les interprètes abordent l'œuvre avec leurs propres vision et bagage. Lorsque questionné sur la façon dont il perçoit son rôle dans l'exposition, un interprète de This Progress à New York déclare: "Je ne suis pas un acteur: je n'invente rien, je parle de moi. Cela n'exclut pas que je joue un certain rôle. Pour moi, ce n'est pas normal d'aborder les gens ainsi [...] je suis un peu acteur forcément ${ }^{47}$ \%. En conséquence, les gens embauchés disposent d'une grande liberté d'action concernant les sujets abordés, comme en témoigne également un interprète de This Situation à Montréal ${ }^{48}$. Cependant, certaines consignes générales sont imposées à tous : interdiction de parler de l'œuvre et d'engager des conversations trop personnelles ou intimes avec les participants.

Qu'en est-il du visiteur? En lui donnant la parole, Sehgal lui permet d'intervenir dans le contenu de la pièce par ses commentaires et son interaction. En discutant avec les interprètes de la pièce et en répondant aux questions qu'il lui pose - ce que Pablo Helguera nomme la "participation créative " ${ }^{49}$-, le sujet occupe une place centrale dans l'œuvre en l'alimentant à intérieur d'une structure définie par l'artiste. Cette notion est centrale chez Sehgal, puisque l'expérience artistique est soumise à des règles précises. Si la structure de la situation et certaines phrases sont prédéterminées par l'artiste, les anecdotes racontées par les interprètes et les réponses fournies par les

47 Entrevue avec Leland De La Durantaye, interprète, le 27 février 2010.

48 Halldór Smith, op. cit.

49 Dans son ouvrage, Pablo Helguera dresse une typologie de la structure participative de l'art socialement engagé dans laquelle figure la participation créative. HELGUERA, Pablo. Education for Socially Engaged Art. New York: Jorge Pinto Books Inc., 2011, p. 15.

50 Si les phrases scénarisées par l'artiste demeureront les mêmes lors des futures présentations de l'œuvre, les réponses et les anecdotes des visiteurs seront différentes à chaque fois. visiteurs varient, ce qui donne lieu à des configurations multiples de l'œuvre ${ }^{50}$. En l'absence du public, il n'y a aucune ouvre à voir et, sans interaction, sa signification demeure cachée. Il n'y a qu'à observer les interprètes en dehors des périodes d'affluence pour s'en convaincre: ceux-ci restent en retrait, postés à différents endroits, en attente.

Ce privilège du participant s'accompagne nécessairement d'une responsabilité tout aussi importante : celle de bien performer et d'offrir sa pleine collaboration, une situation qui peut s'avérer stressante. Il en est d'ailleurs conscient, comme en témoignent les nombreux commentaires publiés sur Internet à la suite de la présentation de This Progress. Alors que certaines personnes ont été amusées par la question posée d'entrée de jeu par l'enfant sur le progrès et par la nature participative de l'œuvre, plusieurs ont été déconcertés, voire contrariés de se faire questionner sur un concept aussi abstrait, sans compter ceux qui ont eu le sentiment de fournir une réponse inadéquate ${ }^{51}$. Le fait d'être interrogé par des inconnus sur une longue période peut s'avérer aussi plaisant qu'inconfortable selon les dispositions, l'état d'esprit ou la personnalité de chacun. En plus d'alimenter et de soutenir une conversation en anglais ${ }^{52}$ avec quatre inconnus, le public devait accomplir un effort physique en gravissant la rotonde spiralée. L'expérience peut ainsi s'avérer complexe et décevante pour l'interprète comme pour le visiteur - voire entraver le bon déroulement de l'œuvre - si ce dernier ne comprend pas le rôle qu'il doit jouer ni ce qu'on attend de lui, laissant ainsi à l'interprète la tâche ardue de relancer la conversation et de pallier les manques.

51 Les commentaires des visiteurs publiés sur Internet dans les différents blogues sont éloquents à cet égard.

52 La langue constitue un frein à la participation, selon nos observations et les entretiens réalisés avec les visiteurs. Le fait que This Progress se déroulait en anglais desservait les touristes. En entrevue, l'artiste nous a révélé que la participation pour la pièce à New York se situait autour de $60 \%$, alors qu'elle était de $90 \%$ lorsque l'exposition a été présentée à Londres en 2007. Cet écart serait attribuable au caractère homogène du public du musée londonien. 
Malgré quelques insatisfactions initiales exprimées par certains, aux dires de Nat Trotman, l'exposition a été favorablement accueillie. Alex Seel, guide au musée Guggenheim, attribue ce succès à la nature personnelle des rapports que l'œuvre instaure (par le biais des interprètes) avec celui qui y participe: "This is real people and they have a real conversation $[\ldots]$ Interpreters always have an amazing story to tell. It's like you are talking to you mom, your dad, your grand-mother $[\ldots]$ ".. ${ }^{53}$. Étant donné que les interprètes s'adressent directement à eux en leur parlant de sujets qui les touchent, les participants se sentent concernés, interpellés, et se confient naturellement. Cependant, tous ne sont pas à l'aise avec cette formule participative qui peut se révéler contraignante et intrusive pour un public habitué à une relation esthétique avec l'œuvre d'art ${ }^{54}$.

Pour This Situation à Montréal, la relation des visiteurs à l'œuvre a été plus ambiguë, selon nos constats. Si le public était d'emblée pris en charge par un interprète dans This Progress, selon ce que nous avons observé, le lien semblait plus difficile à s'établir avec This Situation, où le caractère intellectuel et philosophique des citations en déstabilisait plusieurs. Le visiteur y jouait tantôt le rôle d'un témoin, tantôt le rôle d'un acteur du tableau vivant qui se déroulait devant lui. Questionnés par les interprètes ("Et vous, qu'en pensez-vous?»), certains se retranchaient dans le mutisme ou affirmaient ne pas vouloir répondre ${ }^{55}$, tandis que d'autres, intimidés d'être la cible de l'attention générale, décidaient tout de même de tenter une réponse. Il faut dire que la situation était pour le moins inusitée: six interprètes déclamant solonnellement des citations philosophiques dont certaines remontent à l'Antiquité et sont parfois si hermétiques qu'elles ne permettent pas au public de se les approprier. C'est à ce moment

53 Entrevue avec Alex Seel, guide, le $1^{\mathrm{er}}$ mars 2010. 54 Une journaliste en témoigne. JOHNSON, Paddy. "Tino Sehgal Doesn't Make Much Progress ». The L Magazine, 23 février 2010. <http://www.thelmagazine.com/ newyork/tino-sehgal-doesnt-make-much-progress/ Content?oid=1550271 > (consulté le 14 mars 2010). 55 Plusieurs raisons motivent ce refus de participer de la part des visiteurs, la plus évidente étant la barrière linguistique. Puisque que les citations étaient déclamées parfois en que le rôle de l'interprète prend tout son sens: par ses connaissances des citations, il doit les rendre intelligibles, user d'exemples tirés de l'actualité afin qu'elles fassent sens pour le public, que ce dernier puisse les «traduire " et en discuter par la suite. C'est, selon Rancière ${ }^{56}$, le travail conjugué des interprètes (conteurs) et des visiteurs (traducteurs) qui provoque l'émancipation.

\section{Une médiation repensée}

Bien qu'axées sur le dialogue et la conversation, This Progress et This Situation représentent un défi supplémentaire pour le participant, puisque aucun outil de médiation ni aucune mise en contexte ne le prépare à ce qu'il va vivre. Désirant laisser les situations parler d'ellesmêmes et les rendre vivantes dans le souvenir de ceux qui les expérimentent, l'artiste a exigé des institutions de ne produire aucune documentation dans le cadre des expositions. Ainsi, aucun cartel, panneau explicatif, texte ou catalogue ne guide la lecture, à l'exception du communiqué de presse disponible sur le site Internet des musées. Il n'était d'ailleurs pas envisageable, pour les commissaires, d'ajouter des outils de médiation dans l'exposition, puisque l'œuvre de Sehgal incarne justement cette rencontre entre l'œuvre (par l'intermédiaire des interprètes) et le public ${ }^{57}$. Au sujet d'une situation similaire, l'artiste affirme:

\section{The exhibition had always been much more about people, that is, visitors, walking through spaces as individuals, being addressed by an art experience as individuals. That is really the innovation of the exhibition, of the public exhibition, without the guided tour. The speci- ficity of an exhibition is that it can single out citizens as individual [...] The exhibition had}

anglais, parfois en français, et que les conversations se déroulaient dans ces deux langues, selon les interprètes impliqués, nous avons observé que la langue présentait un obstacle pour certains. D'autres ont évoqué comme argument l'absence d'opinion sur la question.

56 Voir le chapitre "Le spectateur émancipé " dans RANCIÈRE, Le spectateur émancipé, op. cit., p. 28-29. 57 Entrevue avec Nat Trotman, commissaire, le 27 février 2010 . 


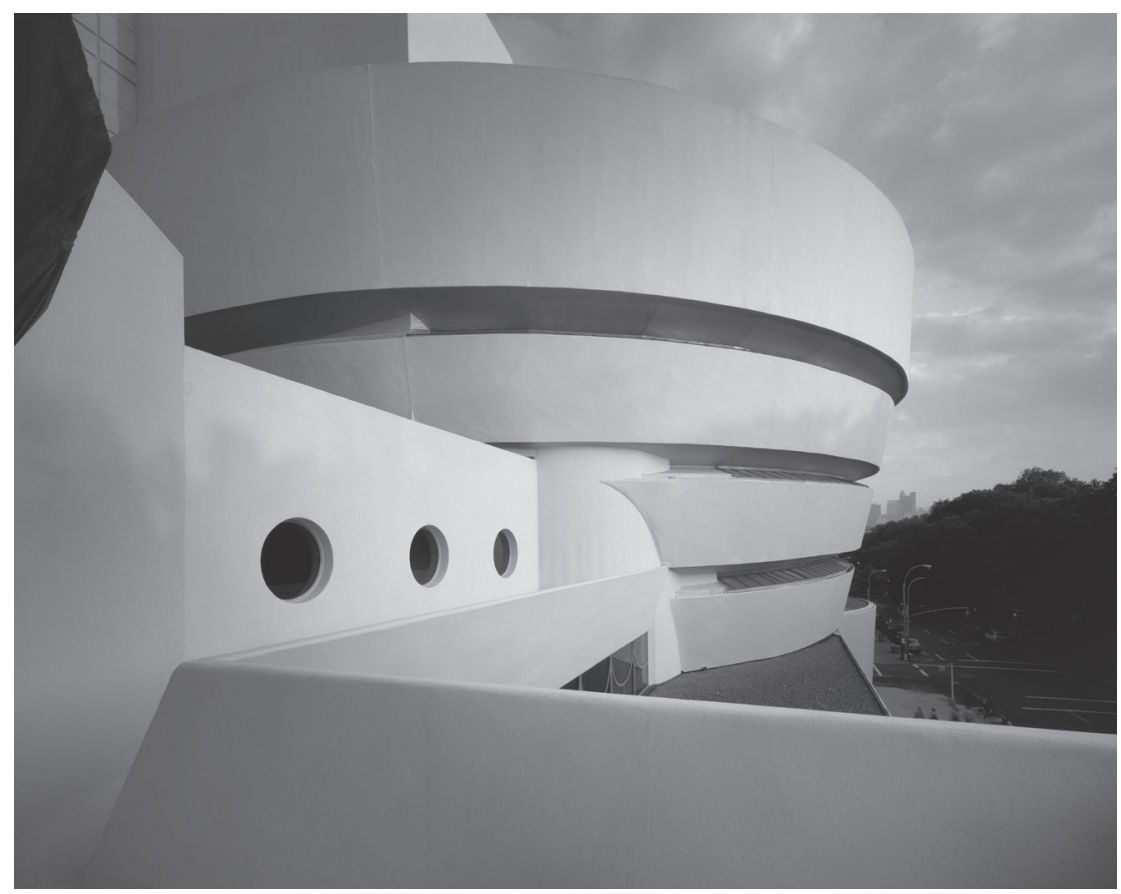

The Solomon R. Guggenheim Museum, New York. ()

The Solomon R. Guggenheim Foundation, New York.

Used with permission [Photographe: David Heald].

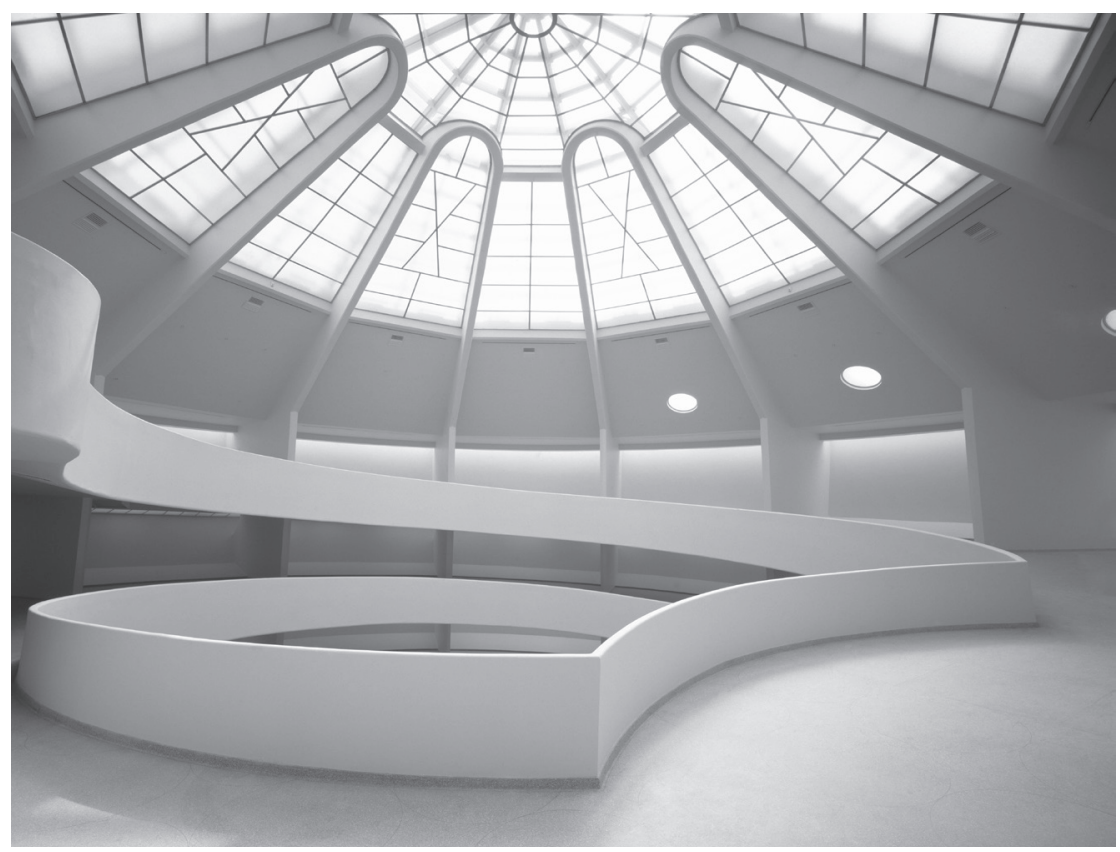

Vue sur la rotonde du musée. The Solomon R. Guggenheim

Museum, New York. (C) The Solomon R. Guggenheim

Foundation, New York.

Used with permission [Photographe: David Heald]. 


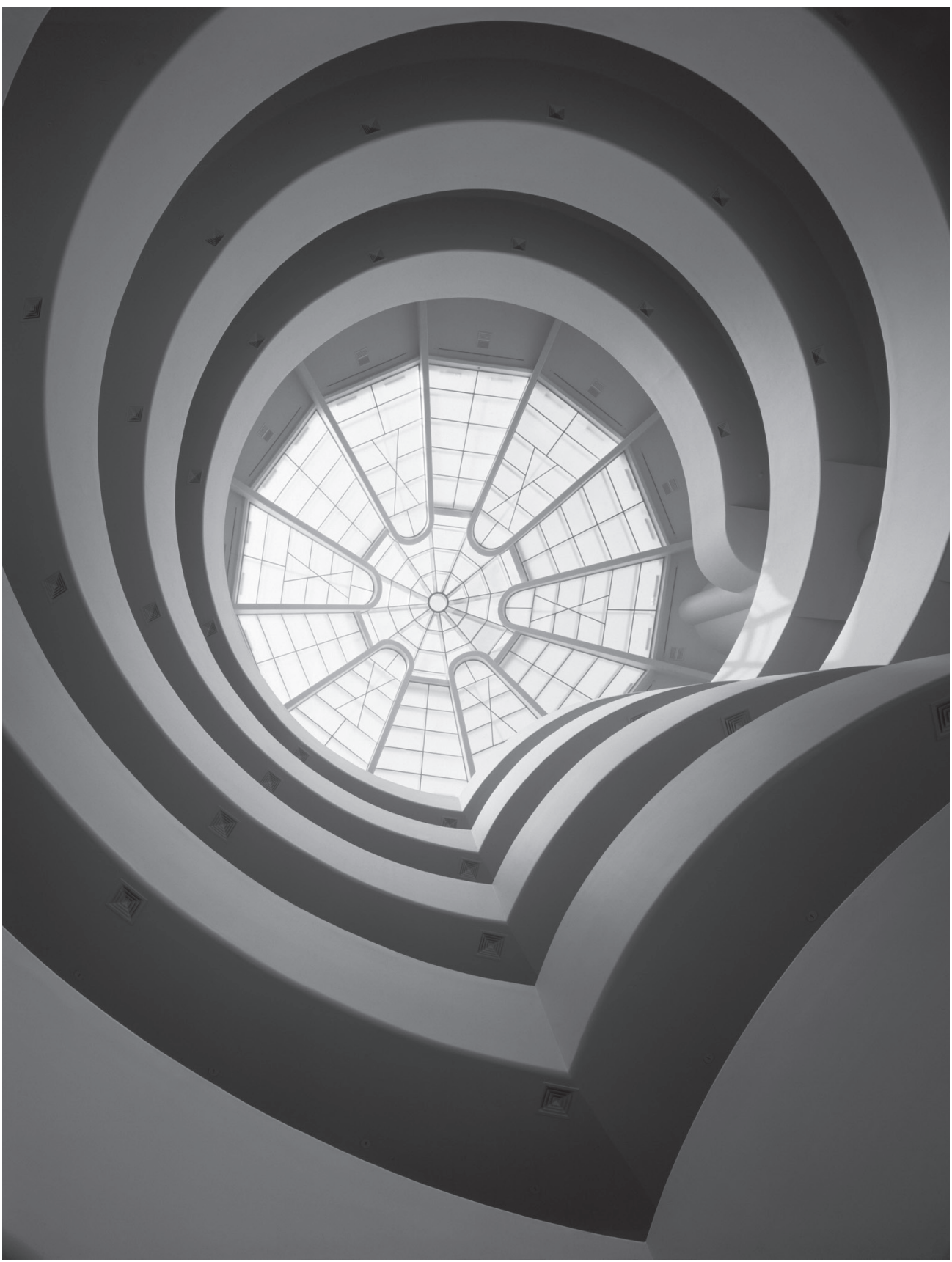

Vue sur la rotonde du musée. The Solomon R. Guggenheim Museum, New York. (c) The Solomon R. Guggenheim

\section{Foundation, New York.}

Used with permission [Photographe: David Heald]. 
always been much more about individuals and about individuals also seeing each other ${ }^{58}$.

Bien que l'œuvre possède en elle-même ce potentiel de médiation ${ }^{59}$, comme le suggère Nat Trotman du Guggenheim, elle s'offre néanmoins sans préparation. Ne sachant pas ce qu'on attend de lui ni comment il doit réagir aux demandes des interprètes, le visiteur peut refuser de collaborer, ce qui est problématique dans une pièce qui mise sur l'interaction, d'où la pertinence et le défi d'ajouter des médiations externes qui appuient la démarche de l'artiste sans l'entraver. Habituellement, le musée new-yorkais mandate des guides - identifiés comme tels - qui circulent dans les salles d'exposition et dont la mission est d'engager la conversation avec les visiteurs ${ }^{60}$. Devant l'impossibilité d'introduire des médiateurs professionnels directement dans l'œuvre sans en perturber le fonctionnement, le musée Guggenheim a donc mis en place une stratégie différente et subtile, avec la complicité du service de l'éducation. Pour This Progress, le rôle des guides consistait à s'assurer que le visiteur emprunte la rampe menant aux étages supérieurs de la rotonde, à l'orienter sans lui donner d'information sur le contenu de l'exposition, puis à lui offrir la possibilité de discuter de son expérience ${ }^{61}$. Postés à différents endroits dans le musée, ils agissaient comme facilitateurs en s'assurant que les gens expérimentent l'œuvre. Contrairement à ce qui se fait habituellement, ces guides n'étaient pas identifiés comme étant des employés du musée : ils portaient leurs propres vêtements, comme les interprètes et les visiteurs, ce qui leur permettait d'exercer leur travail en toute discrétion. Leur rôle n'était pas d'expliquer le sens de l'œuvre ou de servir d'intermédiaire entre l'artiste et le public selon une conception plus traditionnelle de la médiation défendue

58 VON HANTELMANN, "Objects and Situation in the Works of Tino Sehgal ", op. cit., p. 170.

59 Malgré le fait que l'interprète introduise le visiteur à l'œuvre, il n'agirait pas comme médiateur, malgré ce que nous pourrions croire, car il présente l'œuvre sans toutefois en faciliter la compréhension, contrairement à ce que ferait un médiateur.

60 Entrevue avec Alex Seel, guide, le $1^{\mathrm{er}}$ mars 2010.

61 Entrevue avec Nat Trotman, commissaire, le 27 février 2010. notamment par Bruno Latour ${ }^{62}$, mais plutôt d'inciter celui-ci à expérimenter la pièce, en plus de lui donner l'occasion de s'exprimer sur l'œuvre. Nous n'avons pas constaté de pratique similaire au Musée d'art contemporain; la médiation se faisait plutôt à la demande des groupes scolaires ${ }^{63}$.

En plus de l'exposition présentée à New York, le musée a entrepris deux activités, soit la conférence "How To Do Things with Art » sur la dématérialisation de l'objet, par l'historienne de l'art Dorothea von Hantelmann, et le forum virtuel «Material Worth" avec des spécialistes en histoire de l'art. À Montréal, deux rencontres ont été organisées, dont une sur le processus de création de Sehgal où le public était invité à rencontrer la commissaire Lesley Johnstone et le producteur de l'exposition, Asad Reza. Dans le cas de pratiques participatives, la présentation d'activités en soutien aux expositions pourrait permettre au public de mieux saisir les enjeux soulevés par ces pratiques et faciliter leur réception dans un contexte muséal.

\section{Conclusion: le musée comme lieu politique et social}

La présentation d'œuvres dialogiques obligerait l'institution à reconsidérer non seulement sa façon d'envisager l'exposition, mais également son mandat éducatif. Comme le suggère Sally Tenant, chef des programmes à la Serpentine Gallery (Londres), il conviendrait de repenser le musée de l'intérieur et de développer de nouveaux modèles d'éducation basés sur une approche intégrée des services de l'éducation et de la conservation, c'est-à-dire qui favorise les rapprochements des deux disciplines à l'image du New Institutionalism ${ }^{64}$. Selon Simon Sheikh,

62 CAILLET, Élisabeth. "L'ambiguité de la médiation culturelle: entre savoir et présence ". Publics et Musée, no 6, 1995, p. 59-60.

63 Entrevue avec Lesley Johnstone, commissaire, le 24 avril 2013.

64 TENANT, Sally. " Experiment in Integrated Programming ". In. O'NEILL, Paul et Mick WILSON (dir.). Curating and the Educational Turn. Londres : Open Editions, 2010, p. 188 et 190 . 
commissaire et critique d'art, instituer de nouvelles pratiques moins disciplinaires et pédagogiques permettrait également au musée de se distancer du modèle d'éducation traditionnel (basé sur le contenu) pour miser sur l'expérience (le processus) et ainsi amorcer un virage pédagogique favorisant l'émancipation du visiteur $^{65}$. This Situation encouragerait selon nous cette approche multidisciplinaire en mettant en présence des visiteurs de tous les horizons et des interprètes dépositaires des idées des plus grands philosophes. Ce faisant, l'œuvre de Sehgal pourrait également servir de prétexte pour susciter la réflexion sur des thèmes abordés par l'œuvre lors de discussions informelles en dehors de l'exposition. Avec une œuvre axée sur la conversation, le musée deviendrait ainsi un lieu d'échange d'idées, d'opinions, de souvenirs, sans que l'objet matériel ne soit nécessairement présent. De nouvelles perspectives s'offrent ainsi à l'institution muséale qui pourrait profiter d'une telle opportunité pour créer des liens plus étroits avec sa communauté, en réaffirmant son rôle social tant à l'intérieur qu'en dehors de ses murs ${ }^{66}$. C'est ce que permet l'œuvre de Sehgal au musée: un espace propice à l'échange, au dialogue, à la réflexion, et une expérience radicale qui dépasse largement l'expérience esthétique ou éducative de l'art, ce à quoi adhère Pablo Helguera: " what we truly learn is not an abstract concept that is bestowed upon us, but the personal meaning that we construct on our own either by conversation with others or with ourselves " ${ }^{67}$. À notre sens, This Progress s'inscrirait ainsi dans une démarche démocratique d'un "partage esthétique " qui s'expérimente avec l'autre dans l'« événementialité » de la rencontre, selon la conception de la médiation défendue par Rancière $^{68}$. Ce faisant, le destinataire de l'œuvre participerait, avec l'artiste, à la coproduction du sens de l'œuvre et à la constitution de son discours par son interprétation. Selon Rancière, “ [c]'est dans ce pouvoir d'associer

65 SHEIKH, Simon. « Letter to Jane (Investigation of a Function) ». In. O'NEILL et WILSON, op.cit., p. 74-75. 66 Nous pensons notamment aux activités muséales présentées dans l'espace public ou faisant appel aux médias sociaux.

67 Citation de Pablo Helguera. Voir HELGUERA, « Is It Art or Is It Progress? ", op. cit. et de dissocier que réside l'émancipation du spectateur ", c'est-à-dire dans sa capacité à faire le lien entre ce qu'il connaît à ce qu'il ignore ${ }^{69}$.

Ce faisant, les situations de Sehgal réhabiliteraient la tradition orale dans un musée d'art moderne consacré aux œuvres matérielles. Puisque l'artiste n'autorise aucune documentation sur ses œuvres et qu'il est par conséquent impossible de les filmer ou les photographier, celles-ci ne peuvent qu'être transmises oralement, en faisant appel aux souvenirs et aux témoignages des gens qui les ont vécues. À l'image des musées d'histoire et d'ethnologie qui transmettent des récits de vie en donnant accès à l'intimité des gens par le biais de témoignages filmés ou écrits, les œuvres de Sehgal favoriseraient cette proximité entre les interprètes et le public en abolissant toute distance entre eux ${ }^{70}$. Ainsi, le visiteur n'agirait pas comme un observateur disposant d'un recul historique, mais plutôt comme un participant qui alimente in preasentia la discussion par son récit de vie, son histoire personnelle et ses opinions. Ces situations deviendraient conséquemment des témoins privilégiés de l'époque actuelle et de la façon dont les personnes qui y sont impliquées envisagent la société. Durant les six semaines, soit la durée d'une exposition, elles transformeraient, selon nous, le musée en agora et en espace politique. En donnant une voix au visiteur et en le reconnaissant dans son individualité, les œuvres de Sehgal, par les discussions qui s'y déroulent, les idées qui s'y échangent et qui s'y confrontent, rendraient l'institution vivante, ce que confirme la réception critique de l'exposition.

Par leur nature participative, This Progress et This Situation incarnent parfaitement cette dualité dialogique qui habite le musée, à la fois dans le processus de l'œuvre centré sur la conversation et dans la collaboration entre les acteurs impliqués dans l'exposition. En

68 CAILLET, «L'ambiguïté de la médiation culturelle: entre savoir et présence ", op. cit., p. 59-60. 69 RANCIÈRE, chapitre "Le spectateur émancipé ", op. cit., p. 23 et 28 .

70 Avec This Situation toutefois, les citations peuvent être intimidantes et contribuer à tenir le public à l'écart. 
esthétisant une expérience sociale et politique, les pièces de Sehgal modifieraient non seulement le rôle du public, mais celui des professionnels du musée agissant comme organisateurs ou intermédiaires, en soutien à l'artiste qui délègue une partie de sa responsabilité au public et ainsi aux interprètes. Si de nombreux théoriciens ont récemment soulevé les impacts sociaux et politiques positifs des pratiques dialogiques présentées dans l'espace public ${ }^{71}$, ces œuvres réhabiliteraient l'expérience sociale dans un musée d'art en positionnant le visiteur comme acteur et témoin de l'œuvre, bien qu'aucune trace physique ni de son passage ni de l'œuvre ne subsiste une fois la présentation terminée. Une question mérite qu'on s'y attarde: le musée d'art contemporain du XXI ${ }^{\mathrm{e}}$ siècle pourrait-il être envisagé comme un lieu de collaboration, d'expérimentation et de dialogue entre les œuvres, les professionnels, les publics et les artistes, comme l'ont démontré le musée Guggenheim de New York et le Musée d'art contemporain de Montréal? 
Isabelle Riendeau, translated by Olga Gorban

\section{Conversations and Collaboration at the Museum: The Case of Tino Sehgal}

For the last twenty years there has been a growing presence on the contemporary art scene as well as at the museum of situational, relational and dialogical works of art, i.e. of experimental practices which rely on human relations and which actively engage the visitors by including them into a creative process.

Accustomed to exhibit, to collect, to preserve works and to educate the visitors, the museum becomes, when it exhibits these intangible forms of art, a meeting place for the works, the artists, the professionals and the visitors. Although the communication and the dialogue are taking place at the museum - its educational mission and its concern about various public witness it - through these works the relation and the conversation with the visitor become the very foundation of the artistic practice, sometimes putting the object in the background.

The presentation by the museum of process-based and living works focused on the encounter does not go without bringing about the important changes not only for the professionals, but for all those in touch with these works, i.e. the artist and the visitor, in particular with regard to the roles of each, to the organisational and relational dynamics at the museum.

With reference to this transformation, we should consider the challenges and issues that the reception of these forms of art by a museum institution raises. Within this context, what role should the museum, the artist and the visitor play in these living works? This article addresses these questions in relation to a notably representative work of the integration of these new forms of art by the museum-This Progress by the English artist Tino Sehgal. Exhibited in winter 2010 in the Solomon R. Guggenheim Museum in New-York city on the occasion of its $50^{\text {th }}$ Anniversary, this "constructed situation" challenges the conventions and the foundations of a museum institution in many respects.

Since no documentation (visual and written) or mediation are allowed by the artist within the context of the exhibition, Sehgal's work exists only in time-space continuum of the exhibition and in the memory of those who saw it and, especially, experimented it. Relying exclusively on the action of the interpreters who put life into the work of art and the visitors who participate in it, This Progress brought a significant challenge to the New-York Museum.

The results that we present in this article come mainly from a field study - including observation sessions and interviews - performed as part of our doctoral thesis during the presentation of Tino Sehgal's exhibition in the Guggenheim Museum in 2010. First, we look at the significant 
work of coordination and cooperation needed by the Museum in order to exhibit this considerable work featuring more than 100 interpreters. Then we'll examine the functioning of this special exhibition, its dialogical and discursive structures as well as the role and the experience of each person involved, from the curator to the visitor, have had.

This analysis highlights the necessity for the museum to reconsider at the same time its concept of the exhibition (curatorial practice) to receive and transmit appropriately these forms of experimental art. From the place of exhibition, of memory, of research and of learning, the museum would be considered from now on, like the relational works that it exhibits, as a place of cooperation, of experimentation and of dialogue among the professionals, the artists and the visitors. 